\title{
Numerical Existence Property and Categories with an Internal Copy
}

\author{
Samuele Maschio
}

\begin{abstract}
We define here a notion of internal copy and of weak internal copy of a category. We will then determine some families of categories having an internal copy or a weak internal copy. We will consider categories of definable classes of first-order theories and we will see that the notion of internal copy is related to the notion of numerical existence property.
\end{abstract}

Mathematics Subject Classification. Primary 18A15; Secondary 03F40, $03 \mathrm{~F} 55$.

Keywords. Internal categories, Existence property, Definable classes, Gödelian encoding.

\section{Introduction}

A category $\mathbb{C}$ can host internal algebraic structures such as monoids, groups, rings, etc. Among these internal algebraic structures there are categories, too. An internal category is defined as an internal graph having a composition arrow and an identity arrow making some diagrams (representing associativity of composition and properties of identities) commute (see e.g. [3]).

An internal category cannot directly be compared with the category in which it lives. However, it can be "externalized" by means of global elements. It is hence natural to compare this externalization with $\mathbb{C}$.

In this paper we deal with the question whether there exist categories $\mathbb{C}$ having an internal copy, that is an internal category in $\mathbb{C}$ of which the externalization is isomorphic to $\mathbb{C}$ itself. We will show that one can produce examples of a weakening of this notion by considering some categories of definable classes of first-order theories. A metaproperty called numerical existence property will play a crucial role in this case. Finally, we will produce an example of a category with an internal copy in the strong sense. 
Notational conventions In this paper we adopt the following convention: whenever we say that $\mathbb{C}$ is a finitely complete category, we mean that $\mathbb{C}$ is endowed with a distinct terminal object 1 and with an explicit choice of pullbacks $\left(\mathrm{Pb}(f, g), \pi_{0}^{f, g}, \pi_{1}^{f, g}\right)$ for every pair of arrows $f$ and $g$ having the same codomain. In this case, if $k_{0}$ and $k_{1}$ are arrows in $\mathbb{C}$ such that $f \circ k_{0}=g \circ k_{1}$, then we denote by $\left\langle k_{0}, k_{1}\right\rangle_{f, g}$ the only arrow from $\partial_{0}\left(k_{0}\right)=\partial_{0}\left(k_{1}\right)$ to $\mathrm{Pb}(f, g)$ such that $\pi_{i}^{f, g} \circ\left\langle k_{0}, k_{1}\right\rangle_{f, g}=k_{i}$ for $i=0,1$. If $!_{A}: A \rightarrow 1$ and $!_{B}: B \rightarrow 1$ are the unique arrows from $A$ and $B$, respectively, to the terminal object 1 , then we denote with $A \times B$ the object $\mathrm{Pb}\left(!_{A}, !_{B}\right)$, with $\pi_{i}^{A, B}$ the arrow $\pi_{i}^{!_{A}, !_{B}}$ for $i=0,1$ and with $\langle f, g\rangle_{A, B}$ the arrow $\langle f, g\rangle_{!_{A}, !_{B}}$. As usual, we define $\Delta_{A}$ as $\left\langle\operatorname{id}_{A}, \operatorname{id}_{A}\right\rangle_{A, A}$ and $\mathrm{tw}_{A}$ as $\left\langle\pi_{1}^{A, A}, \pi_{0}^{A, A}\right\rangle_{A, A}$. We will omit the subscripts and superscripts when they will be clear from the context.

\section{Internal Copies and Extensions of a Category}

Definition 2.1. An internal category $\Gamma$ in a finitely complete category $\mathbb{C}$ is a 6-uple $\left(\Gamma_{0}, \Gamma_{1}, \delta_{0}, \delta_{1}\right.$, ID,$\left.\square\right)$ where $\Gamma_{0}, \Gamma_{1}$ are objects of $\mathbb{C}$ and $\delta_{0}, \delta_{1}: \Gamma_{1} \rightarrow \Gamma_{0}$, ID $: \Gamma_{0} \rightarrow \Gamma_{1}$ and $\square: \operatorname{Pb}\left(\delta_{1}, \delta_{0}\right) \rightarrow \Gamma_{1}$ are arrows of $\mathbb{C}$ such that

1. $\delta_{i} \circ \mathrm{ID}=\mathrm{id}_{\Gamma_{0}}$ for $i=0,1$;

2. $\delta_{i} \circ \square=\delta_{i} \circ \pi_{i}$ for $i=0,1$;

3. $\square \circ\left\langle\mathrm{ID} \circ \delta_{0}, \mathrm{id}_{\Gamma_{1}}\right\rangle=\square \circ\left\langle\mathrm{id}_{\Gamma_{1}}, \mathrm{ID} \circ \delta_{1}\right\rangle=\mathrm{id}_{\Gamma_{1}}$;

4. $\square \circ\left\langle\pi_{0}, \square \circ \pi_{1}\right\rangle=\square \circ\left\langle\square \circ \pi_{0}, \pi_{1}\right\rangle \circ\left\langle\left\langle\pi_{0}, \pi_{0} \circ \pi_{1}\right\rangle, \pi_{1} \circ \pi_{1}\right\rangle$.

Definition 2.2. If $\Gamma$ is an internal category of a finitely complete category $\mathbb{C}$, then its externalization is the category $\operatorname{Ext}_{\mathbb{C}}(\Gamma)$ defined as follows:

1. $\operatorname{Obj}\left(\operatorname{Ext}_{\mathbb{C}}(\Gamma)\right):=\operatorname{Hom}_{\mathbb{C}}\left(1, \Gamma_{0}\right)$;

2. $\operatorname{Arr}\left(\operatorname{Ext}_{\mathbb{C}}(\Gamma)\right):=\operatorname{Hom}_{\mathbb{C}}\left(1, \Gamma_{1}\right)$;

3. $\partial_{i}(f):=\delta_{i} \circ f$ for $i=0,1$ for morphisms $f$ from 1 to $\Gamma_{1}$;

4. $\mathrm{id}_{A}:=\mathrm{ID} \circ A$ for morphisms $A$ from 1 to $\Gamma_{0}$;

5. $g \circ f:=\square \circ\langle f, g\rangle$ for morphisms $f, g$ from 1 to $\Gamma_{1}$ such that $\partial_{1}(f)=\partial_{0}(g)$.

Definition 2.3. Let $\mathbb{C}$ be a finitely complete category. An internal copy of $\mathbb{C}$ is a pair $(\Gamma, \mathbf{I})$ consisting of an internal category $\Gamma$ and an isomorphism $\mathbf{I}$ : $\operatorname{Ext}_{\mathbb{C}}(\Gamma) \rightarrow \mathbb{C}$.

Let us first consider some trivial cases. Let $\mathbb{C}$ be a finitely complete category having an internal copy with $\operatorname{Arr}(\mathbb{C})$ finite; then $\mathbb{C}$ is the trivial category. Indeed, if $\operatorname{Arr}(\mathbb{C})$ is finite, then from $\left|\operatorname{Hom}_{\mathbb{C}}\left(1, \Gamma_{1}\right)\right|=|\operatorname{Arr}(\mathbb{C})|$, it follows that $\operatorname{Hom}_{\mathbb{C}}\left(1, \Gamma_{1}\right)=\operatorname{Arr}(\mathbb{C})$. Since $\operatorname{id}_{\Gamma_{1}} \in \operatorname{Arr}(\mathbb{C})$, we conclude that $1=\Gamma_{1}$ and thus $\mathbb{C}$ has only one object and this object is terminal. Hence $\mathbb{C}$ is the trivial category. Moreover, no non-trivial preorder has an internal copy: if $\mathbb{C}$ is a preorder with an internal copy $\Gamma$, then, since $\operatorname{Hom}_{\mathbb{C}}\left(1, \Gamma_{1}\right)$ has at most one element, $\mathbb{C}$ has at most one arrow. But, having an internal category, $\mathbb{C}$ has at least one arrow. Thus $\mathbb{C}$ is the trivial category.

Obviously, also every locally small category which is not small (e.g. Set, Grp...) cannot have an internal copy. 
We can weaken the notion of internal copy by relativazing it to a doctrine over $\mathbb{C}$. If $\mathbf{p}: \mathbb{C}^{o p} \rightarrow$ InfSL is a (primary) doctrine, that is a contravariant functor from $\mathbb{C}$ to the category of inf-semilattices, we can consider the internal categories in the base category $\mathcal{Q}_{\mathbf{p}}$ of its elementary quotient completion (see $[5])$. The objects of this category are pairs $(I, \rho)$ where $I$ is an object of $\mathbb{C}$ and $\rho \in \mathbf{p}(I \times I)$ is a $\mathbf{p}$-equivalence relation on it, that is

1. $\mathbf{p}\left(\Delta_{I}\right)(\rho)=\top$ in $\mathbf{p}(I)$;

2. $\mathbf{p}\left(\operatorname{tw}_{I}\right)(\rho)=\rho$ in $\mathbf{p}(I \times I)$;

3. $\left.\mathbf{p}\left(\left\langle\pi_{1}, \pi_{2}\right\rangle\right)(\rho) \wedge \mathbf{p}\left\langle\pi_{2}, \pi_{3}\right\rangle\right)(\rho) \leq \mathbf{p}\left(\left\langle\pi_{1}, \pi_{3}\right\rangle\right)(\rho)$ in $\mathbf{p}(I \times I \times I)$.

An arrow from $(I, \rho)$ to $(J, \eta)$ is an equivalence class $[f]$ of arrows $f: I \rightarrow J$ of $\mathbb{C}$ such that $\rho \leq \mathbf{p}(f \times f)(\eta)$ with respect to the equivalence relation for which $f$ and $g$ are equivalent if and only if $\rho \leq \mathbf{p}(f \times g)(\eta)$.

If $\mathbb{C}$ is finitely complete, then $\mathcal{Q}_{\mathrm{p}}$ has all finite products. In particular, a terminal object in $\mathcal{Q}_{\mathbf{p}}$ is given by the pair $(1, \top)$.

Thus for every $(I, \rho)$ in $\mathcal{Q}_{\mathbf{p}}$,

$$
\operatorname{Hom}_{\mathcal{Q}_{\mathbf{p}}}((1, \top),(I, \rho))=\operatorname{Hom}_{\mathbb{C}}(1, I) / \sim_{\rho}
$$

where $f \sim \rho g$ if and only if $\top \leq \mathbf{p}(\langle f, g\rangle)(\rho)$.

Among the doctrines over $\mathbb{C}$ there is in particular the subobject doctrine. We can hence give the following definition:

Definition 2.4. Let $\mathbb{C}$ be a finitely complete category with a primary doctrine $\mathbf{p}$ over it such that $\mathcal{Q}_{\mathbf{p}}$ is finitely complete. A p-internal copy of $\mathbb{C}$ is a pair $(\Gamma, \mathbf{I})$ consisting of an internal category $\Gamma$ of $\mathcal{Q}_{\mathbf{p}}$ and an isomorphism $\mathbf{I}: \operatorname{Ext}_{\mathcal{Q}_{\mathbf{p}}}(\Gamma) \rightarrow$ C. A weak internal copy of a finitely complete category $\mathbb{C}$ is a $\mathbf{S u b} \mathbf{b}_{\mathbb{C} \text {-internal }}$ copy of $\mathbb{C}^{1}$

If the doctrine $\mathbf{p}$ is elementary (see [5]), then one can define a functor $\nabla$ from $\mathbb{C}$ to $\mathcal{Q}_{\mathbf{p}}$ sending each object $I$ to $\left(I, \exists_{\Delta_{I}}(\top)\right.$ ) (where $\exists_{\Delta_{I}}$ is left adjoint to $\left.\mathbf{p}\left(\Delta_{I}\right)\right)$ and sending an arrow $f$ to $[f]$. If $\mathbf{p}$ has comprehensive weak equalizers, then $\nabla$ is full and faithful. In this case, as a consequence, if $\mathbb{C}$ has an internal copy, then it has also a $\mathbf{p}$-internal copy. If $\mathbb{C}$ is regular, the subobject functor is an elementary doctrine having comprehensive weak equalizers (see [5]); hence every internal copy of $\mathbb{C}$ is also a weak internal copy.

From the very definition of $\mathbf{p}$-internal copies, it follows that no nontrivial finite category or preorder has a p-internal copy; moreover, obviously, no locally small non-small category has a p-internal copy.

\section{Categories of Definable Classes}

Let $\mathrm{T}$ be a first-order (intuitionistic or classical) theory with equality ${ }^{2}$. Let $x, y, z$ be fixed distinct variables of the language of $\mathrm{T}$. The category of its definable classes $\mathbb{D C}[\mathrm{T}]$ is defined as follows:

\footnotetext{
${ }^{1}$ It is implicit in the definition that if $\mathbb{C}$ has a weak internal copy, then $\mathbf{S u b}_{\mathbb{C}}$ must be a primary doctrine such that $\mathcal{Q}_{\mathbf{S u b}_{\mathbb{C}}}$ is finitely complete.

${ }^{2}$ When we will refer to first-order theories we will always tacitly assume them to be intuitionistic or classical, and primitively recursively axiomatizable.
} 
1. Its objects are formal expressions $\{x \mid \varphi\}$ where $\varphi$ is a formula of T having at most $x$ as free variable; such objects are called definable classes and we write $t \varepsilon\{x \mid \varphi\}$ as a shorthand for $\varphi[t / x] .^{3}$

2. We will identify objects $\{x \mid \varphi\}$ and $\{x \mid \psi\}$ for which $\varphi \vdash_{\mathrm{T}} \psi$ and $\psi \vdash_{\mathrm{T}} \varphi$;

3. An arrow from $\{x \mid \varphi\}$ to $\{x \mid \psi\}$ in $\mathbb{D} \mathbb{C}[\mathbf{T}]$ is a formula $F$ having at most $x$ and $y$ as free variables, such that

(a) $F \vdash_{\mathrm{T}} \varphi \wedge \psi[y / x]$;

(b) $F \wedge F[z / y] \vdash_{\mathrm{T}} y=z$;

(c) $\varphi \vdash_{\mathrm{T}} \exists y F$;

4. We will identify arrows $F$ and $G$ from $\{x \mid \varphi\}$ to $\{x \mid \psi\}$ in $\mathbb{D} \mathbb{C}[\mathrm{T}]$ such that $F \vdash_{\mathrm{T}} G$ and $G \vdash_{\mathrm{T}} F$;

5. The composition of $F:\{x \mid \varphi\} \rightarrow\{x \mid \psi\}$ and $G:\{x \mid \psi\} \rightarrow\{x \mid \rho\}$ is defined as $\exists z(F[z / y] \wedge G[z / x]):\{x \mid \varphi\} \rightarrow\{x \mid \rho\}$

6. The identity arrow for $\{x \mid \varphi\}$ is the arrow $\varphi \wedge x=y$.

The following proposition shows some sufficient conditions which guarantee the category of definable classes of a first-order theory with equality to be finitely complete. The proof is omitted since it consists simply of a verification.

Proposition 3.1. Let $\mathrm{T}$ be a first-order theory with equality.

1. If $\tau$ is a formula with at most $x$ as free variable such that $\mathrm{T} \vdash \exists ! x \tau$, then $\{x \mid \tau\}$ is a terminal object in $\mathbb{D} \mathbb{C}[\mathrm{T}]$. In particular, if the language of $\mathrm{T}$ has a constant $k$, then $\mathbb{D} \mathbb{C}[\mathrm{T}]$ has a terminal object.

2. If $\pi$ is a formula with at most $x, y$ and $z$ as free variables such that

(a) $\pi \wedge \pi\left[y^{\prime} / y, z^{\prime} / z\right] \vdash_{\mathrm{T}} y=y^{\prime} \wedge z=z^{\prime}$

(b) $\pi \wedge \pi\left[x^{\prime} / x\right] \vdash_{\mathrm{T}} x=x^{\prime}$

(c) $\mathrm{\top} \vdash \exists x \pi$

then, for every pair of definable classes $\{x \mid \varphi\}$ and $\{x \mid \psi\}$, the following is a product diagram in $\mathbb{D} \mathbb{C}[\mathrm{T}]$ :

$$
\{x \mid \varphi\}<\stackrel{\pi_{1}}{\longleftarrow}\{x \mid \exists y \exists z(\pi \wedge \varphi[y / x] \wedge \psi[z / x])\} \stackrel{\pi_{2}}{\longrightarrow}\{x \mid \psi\}
$$

where

(a) $\pi_{1}={ }^{\operatorname{def}} \exists z(\pi \wedge \varphi[y / x] \wedge \psi[z / x])$

(b) $\pi_{2}={ }^{\operatorname{def}} \exists z(\pi[z / y, y / z] \wedge \varphi[z / x] \wedge \psi[y / x])$

3. Every pair of parallel arrows $F, G:\{x \mid \varphi\} \rightarrow\{x \mid \psi\}$ in $\mathbb{D} \mathbb{C}[\mathrm{T}]$ has an equalizer $\exists y(F \wedge G) \wedge x=y:\{x \mid \exists y(F \wedge G)\} \rightarrow\{x \mid \varphi\}$.

As a consequence of the proposition above, the categories of definable classes of Peano arithmetics PA and of Heyting arithmetics HA are finitely complete. The same holds for the categories of definable classes of set theories like ZFC, ZF, IZF and CZF.

In $\mathbb{D} \mathbb{C}[\mathrm{PA}]$ and in $\mathbb{D} \mathbb{C}[\mathrm{HA}]$ a terminal object is given by $\{x \mid x=0\}$, while in set theories the definable class $\{x \mid \neg \exists y(y \in x)\}$ is terminal.

\footnotetext{
${ }^{3}$ As usual, when we will write $\varphi[t / x]$ we will assume to first change the bounded instances in the formula $\varphi$ of variables in $t$, using fresh variables.
} 
For binary products, in HA and in PA one can take $\pi$ to be (a formula representing) $x=2^{y}(2 z+1)$; in set theories, one can take $\pi$ to be

$$
\forall u(u \in x \leftrightarrow(\forall v(v \in u \leftrightarrow(v=y \vee v=z)) \vee \forall v(v \in u \leftrightarrow v=y)))
$$

Definition 3.2. We will call a first-order theory with equality $\mathrm{T}$ having formulas $\tau$ and $\pi$ satisfying the properties in items 1 . and 2 . of proposition 3.1 a cartesian first-order theory with equality. Whenever $\mathrm{T}$ is a cartesian first-order theory with equality, we assume that the structure of finitely complete category of $\mathbb{D} \mathbb{C}[\mathcal{T}]$ is the one determined by the constructions in proposition $3.1 .^{4}$

\section{First-Order Theories with Natural Numbers}

Definition 4.1. Let $\mathbb{C}$ be a cartesian category. A parametric natural numbers object is a triple $(\mathrm{N}, \mathrm{z}, \mathrm{s})$ where $\mathrm{N}$ is an object, and $\mathrm{z}: 1 \rightarrow \mathrm{N}$ and $\mathrm{s}: \mathrm{N} \rightarrow \mathrm{N}$ are arrows, such that for every pair of objects $P, Q$ and every pair of arrows $f: P \rightarrow Q$ and $g: Q \rightarrow Q$ there exists a unique arrow $h: P \times \mathrm{N} \rightarrow Q$ making the following diagram commute:

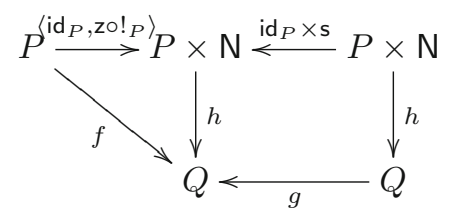

Remark 4.2. In a cartesian category with a parametric natural numbers object, every primitive recursive function between natural numbers can be represented. Indeed, as a consequence of the definition, for every $f: \mathrm{N}^{k} \rightarrow \mathrm{N}$ and $g: \mathrm{N}^{k+2} \rightarrow \mathrm{N}$ there exists a unique arrow rec $[f, g]: \mathrm{N}^{k+1} \rightarrow \mathrm{N}$ making the following diagram commute.

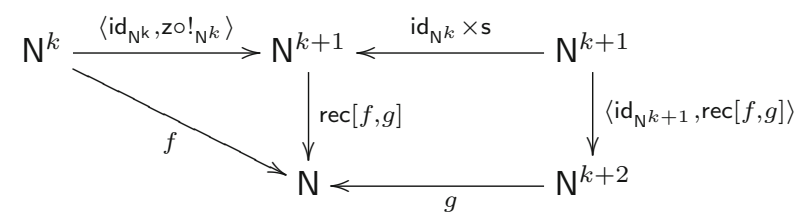

Definition 4.3. A first-order theory with equality $\mathrm{T}$ has natural numbers if its category of definable classes $\mathbb{D} \mathbb{C}(\mathcal{T})$ is cartesian and has a parameterized natural numbers object $(\{x \mid \operatorname{Nat}(x)\}, Z, S)$ for which $S$ is a mono, and $Z$ and $S$ are disjoint. ${ }^{5}$ In this case, for every natural (meta)number $n$ and every formula $\varphi$ we define the formula $\varphi[\underline{n} / x]$ as follows:

1. $\varphi[\underline{0} / x] \equiv{ }^{\operatorname{def}} \exists y \exists x(Z \wedge \varphi[y / x])$

2. $\varphi[\underline{n}+1 / x] \equiv \operatorname{def} \exists y(S[\underline{n} / x] \wedge \varphi[y / x])$

\footnotetext{
${ }^{4}$ Pullbacks can be defined from binary products and equalizers in a canonical way, as shown e.g. in $[2]$

${ }^{5}$ Two monos in a category are disjoint if their pullback is initial.
} 
Set theories ZFC, ZF, IZF and CZF have natural numbers: if we consider the terminal object $\{x \mid \neg \exists z(z \in x)\}$, we take

1. $\operatorname{Nat}(x) \equiv{ }^{\operatorname{def}} \exists w(\operatorname{Trans}(w) \wedge x \in w \wedge$

$$
\forall y(y \in w \rightarrow(\neg \exists z(z \in y) \vee \exists z(z \in w \wedge \operatorname{Succ}(z, y))))
$$

where

(a) $\operatorname{Trans}(w) \equiv{ }^{\operatorname{def}} \forall y \forall z(y \in z \wedge z \in w \rightarrow y \in w)$

(b) $\operatorname{Succ}(z, y) \equiv{ }^{\operatorname{def}} \forall u(u \in y \leftrightarrow(u=z \vee u \in z))$

2. $Z \equiv \operatorname{def} \neg \exists z(z \in x) \wedge x=y$.

3. $S \equiv{ }^{\text {def }} \operatorname{Nat}(x) \wedge \operatorname{Succ}(x, y)$.

The theories of arithmetics PA and HA have natural numbers: Nat $(x) \equiv{ }^{\text {def }}$ $x=x, Z \equiv{ }^{\operatorname{def}} x=y \wedge x=0$ and $S \equiv{ }^{\operatorname{def}} y=\operatorname{succ}(x)$.

\section{Numerical Existence Properties}

Definition 5.1. A first-order theory with equality $T$ having natural numbers has the numerical existence property ( $\mathbf{n E P}$ ) if, for every formula $\varphi$ having at most $x$ as free variable such that $\mathrm{T} \vdash \exists x(\operatorname{Nat}(x) \wedge \varphi)$, there exists a natural (meta)number $n$ such that $\mathrm{T} \vdash \varphi[\underline{n} / x]$.

Numerical existence property $\mathbf{n E P}$ essentially means that if a natural number satisfying a property is proven to exist in $\mathrm{T}$, then a natural (meta)number can be proven to satisfy that property in $\mathrm{T}$.

Peano arithmetic PA, Zermelo-Fraenkel set theory ZF and, in general, classical first-order theories with equality of numbers or sets (if consistent) do not have the numerical existence property. Indeed one can consider an independent sentence $I$ (which exists by Gödel's first incompleteness theorem): clearly $\mathrm{T} \vdash \exists x((x=\underline{0} \wedge \neg I) \vee(x=\underline{1} \wedge I))$ as a consequence of the law of excluded middle; however there cannot be a numeral $\underline{n}$ such that $\mathrm{T} \vdash(\underline{n}=\underline{0} \wedge \neg I) \vee(\underline{n}=$ $\underline{1} \wedge I)$, since in that case $\underline{n}$ would be $\underline{0}$ or $\underline{1}$ and we could hence prove $\neg I$ or $I$ in $\mathrm{T}$.

Heyting arithmetic HA has the numerical existence property: this was proven by means of realizability by Kleene (see [4]). CZF and IZF also have the numerical existence property, as it was proven by Rathjen in [6] and Beeson in [1], respectively.

\section{Internalizing Definable Classes}

Every cartesian first-order theory with equality $\mathrm{T}$ having natural numbers enjoys a primitive recursive Gödelian internal encoding of its syntax by means of natural numbers. We fix such an encoding. We also use, with abuse of notation, symbols for primitive recursive functions between natural numbers (including a primitive recursive bijective encoding of natural numbers $p$ with primitive recursive projections $\mathrm{p}_{1}$ and $\mathrm{p}_{2}$ ), since they can be adequately represented in T. In particular,

1. Every variable $\xi$ in the syntax of $\mathrm{T}$ is encoded by a numeral $\underline{\xi}$; 
2. We use the notation $\widehat{\imath}$ for the encodings of connectives, quantifiers and equality as primitive recursive functions;

3. We use $\operatorname{sub}(x, y, z)$ to denote the code of the formula encoded by $x$ in which the variable encoded by $z$ is substituted by the variable encoded by $y$;

4. There is a predicate form $(x)$ expressing the fact that $x$ is the code of a formula of $\mathrm{T}$;

5. There is a predicate free $(y, x)$ expressing the fact that $y$ is the code of a variable which is free in the formula encoded by $x$;

6. There is a predicate $\operatorname{pf}(u, x, y)$ expressing the fact that $u$ is the code of a proof in $\mathrm{T}$ of the formula encoded by $y$ from the assumption encoded by $x$;

7. There is a predicate notocc $(x, y)$ expressing the fact that the variable encoded by $x$ does not occur in the formula encoded by $y$.

8. We write $\operatorname{der}(x, y)$ as an abbreviation for $\exists u(\operatorname{pf}(u, x, y))$.

One can hence define some formulas which will be helpful in the following sections:

1. We define the formula $\mathrm{dc}(x)$ as

$\operatorname{form}(x) \wedge \forall y(\operatorname{free}(y, x) \rightarrow y=\underline{x}) \wedge \operatorname{notoccur}(\underline{y}, x) \wedge \operatorname{notoccur}(\underline{z}, x)$

which expresses the fact that $x$ is the code of a formula of $\mathrm{T}$ having at most $x$ as free variable. Here and in what follows we will use the formula notoccur to avoid problems with substitutions.

2. We define $\Delta \Gamma_{0}[\mathrm{~T}]:=\{x \mid \mathrm{dc}(x)\}$, which is an object of $\mathbb{D} \mathbb{C}[\mathrm{T}]$.

3. We define the formula $x \equiv_{0} y$ as $\operatorname{dc}(x) \wedge \mathrm{dc}(y) \wedge \operatorname{der}(x, y) \wedge \operatorname{der}(y, x)$.

4. We define the formula $\operatorname{fr}(x)$ as follows

$$
\begin{array}{r}
\text { form }(x) \wedge \forall z(\operatorname{free}(z, x) \rightarrow z=\underline{x} \vee z=\underline{y}) \wedge \\
\operatorname{notoccur}(\underline{z}, x) \wedge \operatorname{der}(x \widehat{\wedge} \operatorname{sub}(x, \underline{z}, \underline{y}), \underline{y} \widehat{=} \underline{z})
\end{array}
$$

expressing the fact that $x$ is the code of a definable functional relation.

5 . In order to encode the collection of arrows of $\mathbb{D C}[\mathrm{T}]$, we need to take track of their codomains (which cannot be reconstructed otherwise). We hence consider the collection

$$
\Delta \Gamma_{1}[\mathrm{~T}]:=\{x \mid \exists y \exists z(x=\mathrm{p}(y, z) \wedge \operatorname{fr}(y) \wedge \mathrm{dc}(z) \wedge \operatorname{der}(y, \operatorname{sub}(z, \underline{y}, \underline{x})))\}
$$

6 . The formula $x \equiv_{1} y$, defined as follows, expresses the equivalence relation of elements $x \in \Delta \Gamma_{1}[\mathrm{~T}]$ :

$$
x \varepsilon \Delta \Gamma_{1}[\mathrm{~T}] \wedge y \varepsilon \Delta \Gamma_{1}[\mathrm{~T}] \wedge \mathrm{p}_{1}(x) \equiv_{0} \mathrm{p}_{1}(y) \wedge \mathrm{p}_{2}(x) \equiv_{0} \mathrm{p}_{2}(y)
$$

7. We define a domain relation $\delta_{0}(x, y)$ as $x \varepsilon \Delta \Gamma_{1}[\mathrm{~T}] \wedge y=\widehat{\exists} y\left(\mathrm{p}_{1}(x)\right)$;

8. We define a codomain relation $\delta_{1}(x, y)$ as $x \varepsilon \Delta \Gamma_{1}[\mathrm{~T}] \wedge y=\mathrm{p}_{2}(x)$;

9. We define an identity relation $\operatorname{ID}(x, y)$ as $x \varepsilon \Delta \Gamma_{0}[\mathrm{~T}] \wedge y=x \widehat{\wedge}(\underline{x} \widehat{=} \underline{y})$;

10. We define a composition relation $\square(x, y)$ as follows:

$$
\begin{gathered}
\mathrm{p}_{1}(x) \varepsilon \Delta \Gamma_{1}[\mathrm{~T}] \wedge \mathrm{p}_{2}(x) \varepsilon \Delta \Gamma_{1}[\mathrm{~T}] \\
\Lambda \exists z \exists u\left(\delta_{1}\left(p_{1}(x), z\right) \Lambda \delta_{0}\left(p_{2}(x), u\right) \Lambda z \equiv{ }_{0} u\right) \\
\wedge \mathrm{p}_{1}(y)=\widehat{\exists} \underline{z}\left(\operatorname{sub}\left(\mathrm{p}_{1}\left(\mathrm{p}_{1}(x)\right), \underline{z}, \underline{y}\right) \widehat{\wedge} \operatorname{sub}\left(\mathrm{p}_{1}\left(\mathrm{p}_{2}(x)\right), \underline{z}, \underline{x}\right)\right) \wedge \mathrm{p}_{2}(y)=\mathrm{p}_{2}\left(\mathrm{p}_{2}(x)\right)
\end{gathered}
$$




\section{An Example of Category with a Weak Internal Copy}

Exploiting the fact that in a category of definable classes $\mathbb{D C}[\mathrm{T}]$ of a first-order theory with equality, every mono $I \rightarrow\{x \mid \varphi\}$ is isomorphic to one of the form $\psi \wedge x=y:\{x \mid \psi\} \rightarrow\{x \mid \varphi\}$, one can prove the following proposition.

Proposition 7.1. Let $\mathrm{T}$ be a first-order theory with equality. The doctrine of subobjects of $\mathbb{D} \mathbb{C}[\mathrm{T}]$ is naturally isomorphic to the first-order hyperdoctrine $\mathbf{L T}_{\mathrm{T}}$ on $\mathbb{D} \mathbb{C}[\mathrm{T}]$ defined as follows:

1. For every object $\{x \mid \varphi\}$ of $\mathbb{D} \mathbb{C}[\mathbf{T}], \mathbf{L T}_{\mathbf{T}}(\{x \mid \varphi\})$ contains all the formulas $\psi$ of $\mathrm{T}$ having at most $x$ as free variable such that $\psi \vdash_{\mathrm{T}} \varphi$; these formulas are identified when they are provable to be equivalent in $\mathrm{T}$ and they are ordered by $\vdash_{\mathrm{T}}$;

2. For every arrow $F:\{x \mid \varphi\} \rightarrow\{x \mid \psi\}$ of $\mathbb{D} \mathbb{C}[\mathbf{T}]$ and every $\rho$ in $\mathbf{L T}_{\mathbf{T}}(\{x \mid \psi\})$, $\mathbf{L T}_{\mathbf{T}}(F)(\rho) \equiv \operatorname{def} \exists y(F \wedge \rho[y / x])$.

Proof. Each subobject $[J:\{x \mid \psi\} \rightarrow\{x \mid \varphi\}]$ of $\{x \mid \varphi\}$ is sent to $\exists y J[y / x, x / y]$ in $\mathbf{L T}_{\mathbf{T}}(\{x \mid \varphi\})$, while, conversely, each $\rho$ in $\mathbf{L T}_{\mathbf{T}}(\{x \mid \varphi\})$ is sent to the subobject determined by the mono $\rho \wedge x=y:\{x \mid \rho\} \rightarrow\{x \mid \varphi\}$. These functions are well-defined and determine a natural isomorphism between $\mathbf{S u b}_{\mathbb{D C}[\mathbf{T}]}$ and LT $\mathbf{T}_{\mathrm{T}}$

Corollary 7.2. Let $\mathrm{T}$ be a cartesian first-order theory with equality. The category $\mathcal{Q}_{\mathbf{S u b}_{\mathbb{D C}[\mathrm{T}]}}$ is isomorphic to the category $\mathbb{D C}_{q}[\mathrm{~T}]$ of which the objects are pairs $(\{x \mid \varphi\}, R)$ in which $\{x \mid \varphi\}$ is an object of $\mathbb{D C}_{q}[\mathrm{~T}]$ and $R$ is a formula of $\mathrm{T}$ having at most $x, y$ as free variables which is a definable equivalence relation $^{6}$ on the class $\{x \mid \varphi\}$ (we identify objects of which the equivalence relations can be proven to be equivalent in $\mathrm{T})$; and of which an arrow from $(\{x \mid \varphi\}, R)$ to $(\{x \mid \psi\}, S)$ is an equivalence class of arrows $F$ from $\{x \mid \varphi\}$ to $\{x \mid \psi\}$ in $\mathbb{D} \mathbb{C}[\mathrm{T}]$ such that $F \wedge F\left[x^{\prime} / x, y^{\prime} / y\right] \wedge R\left[x^{\prime} / y\right] \vdash_{\mathrm{T}} S\left[y / x, y^{\prime} / y\right]$ with respect to the equivalence relation for which two such arrows are equivalent if and only if $F \wedge G\left[x^{\prime} / x, y^{\prime} / y\right] \wedge R\left[x^{\prime} / y\right] \vdash_{\mathrm{T}} S\left[y / x, y^{\prime} / y\right]$.

Proof. From proposition 7.1, it follows that $\mathcal{Q}_{\mathbf{S u b}_{\mathbb{D C}[\mathrm{T}]}}$ is isomorphic to $\mathcal{Q}_{\mathbf{L T}}$. Moreover, since $\mathrm{T}$ is cartesian, one can define an isomorphism from $\mathcal{Q}_{\mathbf{L T}}$ to $\mathbb{D C}_{q}[\mathrm{~T}]$ by sending each of its objects $(\{x \mid \varphi\}, \rho)$ to

$$
(\{x \mid \varphi\}, \exists z(\pi[z / x, x / y, y / z] \wedge \rho[z / x]))
$$

and each arrow $F$ to itself.

The category $\mathbb{D C}_{q}[\mathrm{~T}]$ is finitely complete and from the very definition of the abbreviations in the previous section, the following proposition holds.

\footnotetext{
${ }^{6}$ We mean here that $R \vdash_{\mathrm{T}} \varphi \wedge \varphi[y / x]$ and $R$ is provable in $\mathrm{T}$ to be reflexive, symmetric and transitive.
} 
Proposition 7.3. Let $\mathrm{T}$ be a cartesian first-order theory with equality and natural numbers. The sestuple

$$
\left(\left(\Delta \Gamma_{0}[\mathrm{~T}], x \equiv_{0} y\right),\left(\Delta \Gamma_{1}[\mathrm{~T}], x \equiv_{1} y\right),\left[\delta_{0}(x, y)\right],\left[\delta_{1}(x, y)\right],[\operatorname{ID}(x, y)],[\square(x, y)]\right)
$$

is an internal category in $\mathbb{D C}_{q}[\mathrm{~T}]$ which we will denote with $\Delta \Gamma[\mathrm{T}]$, provided that we take as pullback of $\left[\delta_{1}(x, y)\right]$ and $\left[\delta_{0}(x, y)\right]$ the definable class defined by the formula consisting of the first two lines in item 10. in the previous section together with the equivalence relation induced by $\equiv_{1}$ on first and on second components, with the projections determined by $\mathrm{p}_{1}$ and $\mathrm{p}_{2}$.

Theorem 7.4. Every category of definable classes of a cartesian first-order theory $\mathrm{T}$ with equality and natural numbers having the numerical existence property has a weak internal copy.

Proof. We can assume $\mathrm{T}$ to be consistent, since otherwise the thesis trivially holds.

As a consequence of the corollary above, it is sufficient to prove that $\operatorname{Ext}_{\mathbb{D C}_{q}[\mathrm{~T}]}(\Delta \Gamma[\mathrm{T}])$ is isomorphic to $\mathbb{D} \mathbb{C}[\mathrm{T}]$. If we denote with $\widehat{\varphi}$ the natural (meta)number encoding the formula $\varphi$ in the Gödelian encoding we considered, we obtain a functor $\mathbf{J}$ from $\mathbb{D} \mathbb{C}[\mathrm{T}]$ to $\operatorname{Ext}_{\mathbb{D C}_{q}[\mathrm{~T}]}(\Delta \Gamma[\mathrm{T}])$ by sending

1. Each object $\{x \mid \varphi\}$ to $[\tau \wedge y=\underline{\hat{\varphi}}] \in \operatorname{Hom}_{\mathbb{D C}_{q}[\mathrm{~T}]}\left(1,\left(\Delta \Gamma_{0}[\mathrm{~T}], x \equiv_{0} y\right)\right)$

2. Each arrow $F$ from $\{x \mid \varphi\}$ to $\{\bar{x} \mid \psi\}$ to

$$
[\tau \wedge y=\mathrm{p}(\underline{\hat{F}}, \underline{\widehat{\psi}})] \in \operatorname{Hom}_{\mathbb{D C}_{q}[\mathrm{~T}]}\left(1,\left(\Delta \Gamma_{1}[\mathrm{~T}], x \equiv_{1} y\right)\right.
$$

Conversely, we define a functor from $\operatorname{Ext}_{\mathbb{D C}_{q}[\mathrm{~T}]}(\Delta \Gamma[\mathrm{T}])$ to $\mathbb{D} \mathbb{C}[\mathrm{T}]$ as follows.

1. If $[F] \in \operatorname{Hom}_{\mathbb{D C}_{q}[\mathrm{~T}]}\left(1,\left(\Delta \Gamma_{0}[\mathrm{~T}], \equiv_{0}\right)\right)$, then $\mathrm{T} \vdash \exists ! y \exists x F$ and $F \vdash_{\mathrm{T}} \operatorname{Nat}(y)$. Thus, by nEP there exists a natural (meta)number $n$ such that $\mathrm{T} \vdash$ $\exists x F[\underline{n} / y]$. Decoding $n$ one can construct a formula $\varphi_{n}$ such that $\widehat{\varphi_{n}}$ is $n$. The definable class $\left\{x \mid \varphi_{n}\right\}$ is the object to which $[F]$ is sent. This application is well-defined, since if $F$ and $G$ represent the same arrow from 1 to $\left(\Delta \Gamma_{0}[\mathrm{~T}], \equiv_{0}\right)$ in $\mathbb{D C}_{q}[\mathrm{~T}]$, and $n$ and $m$ are natural (meta)numbers such that $\mathrm{\top} \vdash \exists x F[\underline{n} / y]$ and $\mathrm{\top} \vdash \exists x G[\underline{m} / y]$, then $\mathrm{\top} \vdash \exists u \operatorname{pf}(u, \underline{n}, \underline{m})$ and $\mathrm{T} \vdash \exists u \operatorname{pf}(u, \underline{m}, \underline{n})$; hence using $\mathbf{n E P}$ we can find natural (meta)numbers $k$ and $h$ such that $\mathrm{T} \vdash \operatorname{pf}(\underline{k}, \underline{n}, \underline{m})$ and $\mathrm{T} \vdash \operatorname{pf}(\underline{h}, \underline{m}, \underline{n})$. Decoding $k$ and $h$ we obtain actual proofs of $\varphi_{n} \vdash_{\mathrm{T}} \varphi_{m}$ and of $\varphi_{m} \vdash_{\mathrm{T}} \varphi_{n}$.

2. We use an analogous procedure to define the functor on arrows, exploiting nEP.

These two functors determine an isomorphism of categories.

\section{The Classical Case}

In case of cartesian classical first-order theories with equality and natural numbers one can define some quotients in $\mathbb{D} \mathbb{C}[\mathrm{T}]$ using the minimum principle which holds for natural numbers: 
Proposition 8.1. If $\mathrm{T}$ is a classical first-order theory with equality and natural numbers, then the full subcategory $\mathbb{D}^{N a t}[\mathrm{~T}]$ of $\mathbb{D} \mathbb{C}[\mathrm{T}]$ of which the objects are those of the form $\{x \mid \varphi\}$ with $\varphi \vdash_{\mathrm{T}} \operatorname{Nat}(x)$ is equivalent to the full subcategory $\mathbb{D C}_{q}^{N a t}[\mathrm{~T}]$ of $\mathbb{D C}_{q}[\mathrm{~T}]$ of which the objects are those of the form $(\{x \mid \varphi\}, R)$ with $\varphi \vdash_{\mathrm{T}} \operatorname{Nat}(x)$.

Proof. One just have to exploit the fact that in $\mathrm{T}$ the minimum principle for natural numbers holds. We define a functor $\mathbf{Q}: \mathbb{D C}_{q}^{N a t}[\mathrm{~T}] \rightarrow \mathbb{D} \mathbb{C}[\mathrm{T}]$ as follows:

1. Every object $(\{x \mid \varphi\}, R)$ in $\mathbb{D C}_{q}^{N a t}[\mathrm{~T}]$ is sent to ${ }^{7}$

$$
\mathbf{Q}(\{x \mid \varphi\}, R):=\{x \mid \varphi(x) \wedge \forall y(R \rightarrow x \leq y)\}
$$

2. Every arrow $[F]:(\{x \mid \varphi\}, R) \rightarrow(\{x \mid \psi\}, S)$ is sent to the arrow

$$
x \varepsilon \mathbf{Q}(\{x \mid \varphi\}, R) \wedge y \varepsilon \mathbf{Q}(\{x \mid \psi\}, S) \wedge \forall y^{\prime}\left(F\left[y^{\prime} / y\right] \rightarrow S\left[y / x, y^{\prime} / y\right]\right) .
$$

Conversely, we can define a functor $\mathbf{Q}^{\prime}: \mathbb{D} \mathbb{C}^{N a t}[\mathrm{~T}] \rightarrow \mathbb{D C}_{q}[\mathrm{~T}]$ as follows: every object $\{x \mid \varphi\}$ in $\mathbb{D}^{N a t}[\mathrm{~T}]$ is sent to $(\{x \mid \varphi\}, \varphi \wedge x=y)$ and every arrow $F$ is sent to $[F]$.

This pair of functors gives rise to an equivalence of categories.

In this case a functor $\mathbf{J}$ can still be defined from $\mathbb{D} \mathbb{C}[T]$ to $\operatorname{Ext}_{\mathbb{D} \mathbb{C}_{q}[T]}(\Delta \Gamma[T])$ exactly as it is done in the first part of the proof of theorem 7.4.

Moreover, since $\Delta \Gamma[T]$ is defined using only formulas regarding natural numbers, it can be seen as an internal category in $\mathbb{D C}_{q}^{N a t}[\mathrm{~T}] .^{8}$ By proposition 8.1 , the category $\mathbb{D} \mathbb{C}_{q}^{N a t}[\mathrm{~T}]$ is equivalent to the full subcategory $\mathbb{D} \mathbb{C}^{N a t}[\mathrm{~T}]$ of $\mathbb{D} \mathbb{C}[\mathrm{T}]$. Thus $\Delta \Gamma[\mathrm{T}]$ can be turned into an internal category of $\mathbb{D} \mathbb{C}[\mathrm{T}]$ of which the externalization is isomorphic to $\operatorname{Ext}_{\mathbb{D} \mathbb{C}_{q}[T]}(\Delta \Gamma[T])$.

This would make this internal category a possible candidate to be an internal copy of $\mathbb{D} \mathbb{C}[\mathrm{T}]$. However, if $\mathrm{T}$ is consistent, the lack of the numerical existence property is an obstacle to prove that $\mathbf{J}$ is an isomorphism. Indeed, we cannot turn a global element of $\Delta \Gamma_{0}$, which gives us a "definable" code for a formula, in a natural (meta)number from which we can construct an actual formula of $\mathbf{T}$. Faithfulness of $\mathbf{J}$ and its injectivity on objects are also obstacled by the lack of the numerical existence property: $\mathrm{T}$ could in principle prove the existence of (a code of) a proof of equivalence between two (codes of) formulas $\varphi$ and $\psi$, also in cases in which we cannot (meta)prove there is an actual proof for such an equivalence.

\section{An Example of Category with an Internal Copy}

Theorem 9.1. Let $\mathrm{T}$ be a cartesian first-order theory with equality having natural numbers and having the numerical existence property. Then $\mathbb{D C}_{q}[\mathrm{~T}]$ has an internal copy.

\footnotetext{
${ }^{7} x \leq y$ denotes the order relation between natural numbers which can be defined in $\mathrm{T}$. ${ }^{8} \mathbb{D} \mathbb{C}_{q}^{\text {Nat }}[\mathrm{T}]$ is a finitely complete category and its embedding in $\mathbb{D} \mathbb{C}_{q}[\mathrm{~T}]$ preserves finite limits, the same holds for $\mathbb{D} \mathbb{C}^{N a t}[T]$ with respect to $\mathbb{D} \mathbb{C}[\mathbf{T}]$.
} 
Proof. One can modify $\Delta \Gamma[\mathrm{T}]$ and obtain an internal category $\Delta \Gamma^{q}[\mathrm{~T}]$ by adding the encoding of equivalence relations in the formulas defining the class of (codes for) objects and the conditions about their preservations in the class of (codes for) arrows (together with a track of both domain and codomain), and by adequately modifying the internal equivalence relations on them. Numerical existence property will allow to establish the isomorphism between $\mathbb{D C}_{q}[\mathrm{~T}]$ and $\operatorname{Ext}\left(\Delta \Gamma^{q}[\mathrm{~T}]\right)$

\section{Acknowledgements}

Open access funding provided by Università degli Studi di Padova within the CRUI-CARE Agreement. The author would like to thank Francesco Ciraulo, Fabio Pasquali and Giovanni Sambin for fruitful discussions on the topic of the present paper.

Open Access. This article is licensed under a Creative Commons Attribution 4.0 International License, which permits use, sharing, adaptation, distribution and reproduction in any medium or format, as long as you give appropriate credit to the original author(s) and the source, provide a link to the Creative Commons licence, and indicate if changes were made. The images or other third party material in this article are included in the article's Creative Commons licence, unless indicated otherwise in a credit line to the material. If material is not included in the article's Creative Commons licence and your intended use is not permitted by statutory regulation or exceeds the permitted use, you will need to obtain permission directly from the copyright holder. To view a copy of this licence, visit http://creativecommons. org/licenses/by/4.0/.

Publisher's Note Springer Nature remains neutral with regard to jurisdictional claims in published maps and institutional affiliations.

\section{References}

[1] Beeson, M.: Continuity in intuitionistic set theories. In: Boffa, M., van Dalen, D., Lascar, D., Smiley, J. (eds.) Logic Colloquium '78. North-Holland, Amsterdam (1979)

[2] Borceux. F.: Handbook of Categorical Algebra. 3, volume 52 of Encyclopedia of Mathematics and Its Applications. Categories of Sheaves. Cambridge University Press, Cambridge (1994)

[3] Johnstone, P.: Topos Theory. Academic Press, Cambridge (1977)

[4] Kleene, S.C.: On the interpretation of intuitionistic number theory. J. Symbol. Log. 10(4), 109-124 (1945)

[5] Maietti, M.E., Rosolini, G.: Elementary quotient completion. Theory Appl. Categor. 27(17), 445-463 (2013)

[6] Rathjen, M.: The disjunction and related properties for constructive ZermeloFraenkel set theory. J. Symbol. Log. 70(4), 1233-1254 (2005) 
Samuele Maschio

Dipartimento di Matematica "Tullio Levi-Civita"

Università degli studi di Padova

Via Trieste, 63

35131 Padua

Italy

e-mail: maschio@math.unipd.it

Received: July 16, 2019.

Accepted: July 13, 2020. 